\title{
Technology of Concrete: Reality and Perspectives
}

ISSN: 2639-0574

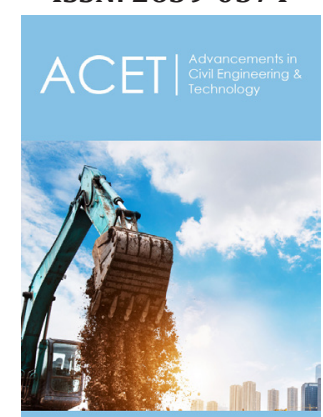

${ }^{* 1}$ Corresponding author: Pshenichny GN, Institute of Construction and Transport Infrastructure FGBOU VO, Russia

Submission: 侮 May 05, 2019

Published: 海 May 20, 2019

Volume 3 - Issue 2

How to cite this article: Pshenichny GN ,Technology of Concrete: Reality and Perspectives.Adv Civil Eng Tech. 3(2). ACET.000558.2019.

DOI: 10.31031/ACET.2019.03.000558

Copyright@ Pshenichny GN, This article is distributed under the terms of the Creative Commons Attribution 4.0 International License, which permits unrestricted use and redistribution provided that the original author and source are credited.

\author{
Pshenichny GN* \\ Institute of Construction and Transport Infrastructure FGBOU VO, Russia
}

\begin{abstract}
The technological process of the production of prefabricated and monolith reinforced concrete products presupposes the use of raw materials with the required characteristics, the use of the rational composition of the concrete mix, its qualitative preparation, problem-free transportation, packing in a form, the careful compaction and ensuring of the favorable conditions for solidification. This is all true. The presented technological sequence is a well-known and generally accepted scheme of normal production. At the same time, it is difficult to call such a process directional. It cannot be directed because after all these required preparatory operations the fundamental aspect the formation of concret's structure and characteristics are left to the concrete itself. In the process of solidification, both positive and negative phenomena are stochastically flowing and superposing on each other. So it is difficult to predict which of them will have a predominant effect on the final product. Thus, only the process can be qualified as directed, which initial stage is under active technological control and accompaniment, consistent with the kinetics of hardening of the Portland cement. As a consequence, the indispensable condition for a directed and meaningful technology is a clear understanding of the physical essence of the conversion of plastic cement mass into stone and a concrete mixture into concrete. The refinement of individual theoretical positions of the hydration and structure-forming problems was the goal of this work.
\end{abstract}

Keywords: Portland cement; Hydration; Concrete; Process staging; Micro-concrete; Surface-active zones; Dump strength; Reliability

\section{Foreword}

The fundamental principle of the concrete technology (reinforced concrete) "with predefined properties" was formulated in the $60 \mathrm{~s}$ of the last century. It says that the production process must be "directed", i.e. by all means coordinated with the mechanism of portland cement hardening. A negative answer to the question whether we have reached this technological "orientation" is obvious after many decades. The existing domestic (threestage) and western (five-stage) clinker cementation hardening models, which describe the development of the process according to the scheme "dissolution-colloidation-crystallization", do not adequately reflect the essence of the phenomenon. The confirmation of the current theoretical uncertainty is the mass of chronic "white spots" in these theories. The ultimate in technological excellence, for example, is the complete dissolution of cement grains, which contradicts the experiment, indicating the invariability of their dimensions, the constancy of the hydrate shell thickness ("hydrated rim" in the terminology of Western researchers) on any (large, medium, small) cement corpuscles. The morphology of hydrated cement grains is not clear. What is this object? Is it an amorphous, crystalline product or some symbiosis? The logic and meaning of the «induction period» are not clear too. Such important moments in practical terms, such as «discontinuity» (according to Kind [1]) and "sawtoothness" (according to Malinina [2]) of strength growth are forgotten. The "wavy" change in the number of properties and phenomena accompanying cement hardening (the chemical composition and alkalinity of the pore fluid, the electrophysical parameters, the humidity state, etc.) is completely inconceivable. The reason of the worsening of the contact zone of concrete and reinforced concrete is mysterious too. The determining characteristic of cement concrete is strength, what looks unconvincing against the background of marked sawtoothness.

Simply put, in the absence of a reliable theoretical foundation, the presence of all these and many other not mentioned problems, it is difficult to talk about the meaningfulness and direction of the technology of concrete and reinforced concrete. An extremely active solid phase and a highly organized fluid determine the intensive flow at the border between electrosurface phenomena that determine the repeatedly increasing and decreasing positive charge 
of hydrating grains. The interaction of a heterogeneous cement system should be considered from the standpoint of the theory of the "activated complex" (according to H Eyring) or the "transition state" (according to M Polanyi), providing for the transition of the system from the initial state to the final form by stage formation in the interphase zone from the elements of interacting components of a certain metastable energy composition with its development and decay.

\section{Stage Surface Hardening of the Portlandcements}

It should be noted that chemically active reagents are water and anhydrous cement minerals (mainly calcium silicates), which have little comparable energy of intermolecular bonds (or the necessary work to separate a object into its constituent parts). Thus, to destroy the hydrogen bonds of water associates (clusters), its molecules or hydroxyl groups, the energy loss of the order of, respectively, 25, 495 and $435 \mathrm{~kJ} / \mathrm{mol}$ is required [3]. The energy of water adsorption (depending on the surface features of the substrate), according to some sources, can reach $160 \mathrm{~kJ} / \mathrm{mol}$. This energy is enough to loosen associated water complexes and redistribute dipoles at the phase boundary, but sure insufficient for the destruction of water molecules (hydroxyl groups) and for appearance of highly reactive particles $\left(\mathrm{OH}^{-}, \mathrm{H}_{3} \mathrm{O}^{+}\right.$, etc.). The destruction of calcium silicates (with a binding energy of $2280 \ldots 3380 \mathrm{~kJ} / \mathrm{mol}$ ) as a result of the adsorption process is impossible. Consequently, the traditional approach, which provides for an immediate hydrolysis (dissolution) of calcium silicates in the moment of mixing cement with water, is completely unfounded.

The noted discrepancy can be solved only from the positions formulated in 1935 and with the noted above theory of the transition "activated complex" or "transition condition". The formation of a transition intermediate energy composition with its subsequent development will make it possible to achieve a state of successful completion of the heterogeneous reaction. In this case, the activation energy can be much less (!) than the brake energy of the initial reagents chemical bonds. Therefore, the interaction of cement with the water is not carried out by secondary and dependent (solvent, hydrolysis, chemisorption and so on) actions, but by priority electrostatic interactions. When the reagents come in contact, blanking of free $\mathrm{CaO}$ with a weak exothermic (especially for stale cements) occurs. Double electrical layer (DEL), is formed on the surface of the cement grains instantaneously. It is not a planar configuration (with a uniformly smeared Helmholtz layer of charges), but in the form of locally dispersed porous (domain) polymolecular aquatic clusters (Figure 1) with a diameter of about $0.5 \mu \mathrm{m}$, which were found by American researchers in the middle of the last century [4].

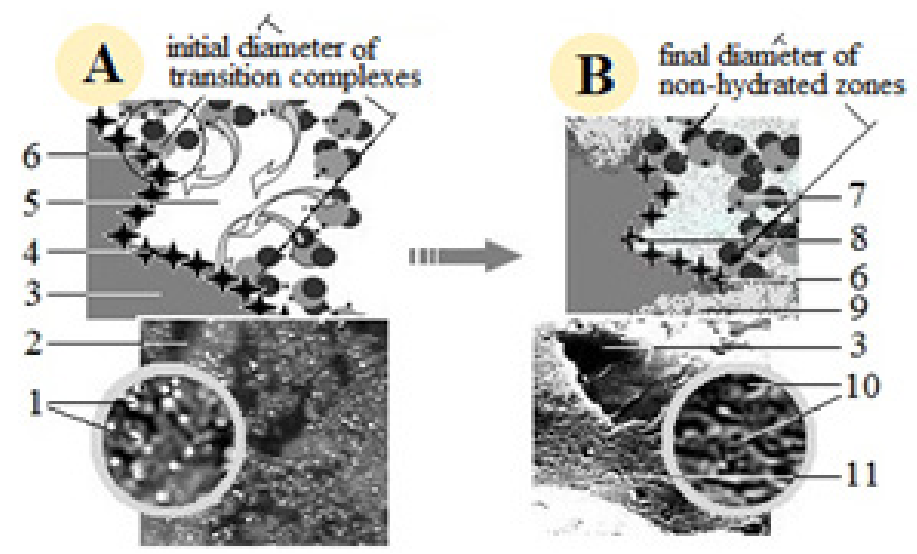

Figure 1: Diagram and general view of the initial (A) and final (B) states of the interacting cement system.

1-transition energy complexes; 2-surface clinker grain; 3-grain array; 4-active center; 5-dipole arch; 6-adsorption center; 7-molecule water; 8-residual active center; 9-hydrate product; 10-residual surface- active zones; 11-hydrated sheath

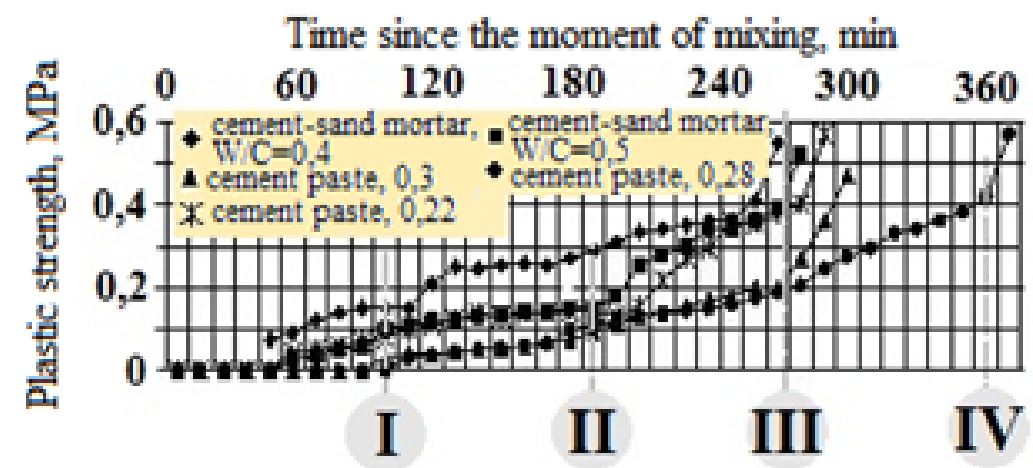

Figure 2: The kinetics of plastic strength is normal hardening cement compositions. 
Thus, the experimentally established fact is that in the moment of contact of cement grains with water on the clinker surface, submitting to the geometry and hydration characteristics of the active center's spatial poly-molecular tent water formations are appearing. Their met stability is due to an excess of unbalanced charges under the poly-molecular arches of the mineral substrate and dynamism (vibration and rotational thermal motion) of the dipoles. The electromagnetic effect of energetically non-saturated active centers of the solid phase causes weakening, consistent failure of hydrogen bonds of clusters, gradual concentration of dipoles in adsorption centers (Figure 1), accumulation of free charge carriers on the surface of cement grains [5]. The rising surface charge (the $\xi$ potential of the spatial double electric layer) is the cause of the stimulation of the adsorbate-adsorbent energy system, which reaches a critical level of activation energy. This energy destroys the hydrogen and chemical bonds of the complex. A part of calcium ions from the structure of minerals is pushed into the fluid, determining the beginning of the main exothermic effect. The appeared highly reactive products of water molecules decomposition (hydronium, modifications of proton hydrates) interact with the silicon-oxygen hydrolysis residues of the solid phase. The resulting hydrate in the form of locally dispersed amorphous clusters covers the surface of clinker particles. The contractional effect both with the intensive consumption of large portions of dipoles by the grains (to form the next energy complex), causes the "propel force" of solidification-the vacuum [6] in intergranular voids. It organizes the cement system, which is usually fixed by the first "jump" in structural strength after an hour and a half interval from the moment from the moment of mixing cement with water (Figure 2).

The appearance of the next activated complex and the abovementioned sequence of the process lead to the formation of a hydrate product in the order of $180 \pm 10 \mathrm{~min}$ (Figure 2 ). So, hydration of cement minerals and the formation of cement stone is the staged process, including alternation of relatively long preparatory (induction) periods and rapid (explosive) moments of the chemise of the phenomenon. The vacuum developing in the system ensures the contraction of cement grains before the appearance of lowstrength hydro silicate contacts with following consolidation and strengthening of the contact zones. As the water chemically binds, the hydrate of active centers, the reducing of the size of the complexes and the surface energy of the grains, accordingly, increases the energy of the hydrogen bonds of adsorbed dipoles, the induction intervals naturally increase, changing with time in hours, days and weeks. The logical conclusion of the process is the formation on the hydrated surface of cement particles locally dispersed relatively stable residual surface-active zones, which can be easily detected by electron microscopy in the form of spherical pores and channels in a hydro silicate mass $0.3 \mu \mathrm{m}$ in diameter and smaller (Figure $1 \& 3$ ). The presence of these non-hydrated zones, with the condensation of highly organized water clusters and their feeding by environmental dipoles is the reason of endless hydration process. The presented stage-surface hardening scheme of Portland cement and materials based on it allows to clarify many of the still existing problems.

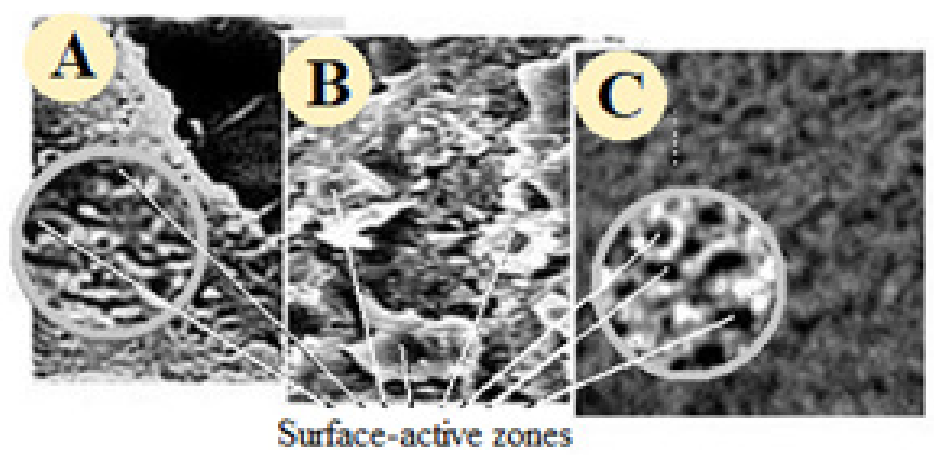

Figure 3: The fragments of the hydrated surface of cement grains of three-month (A, B) and 105-year (C) samples. The Physical Essence of the Characteristics Accompanying the Hardening Portland Cement

\section{The energetics of the process}

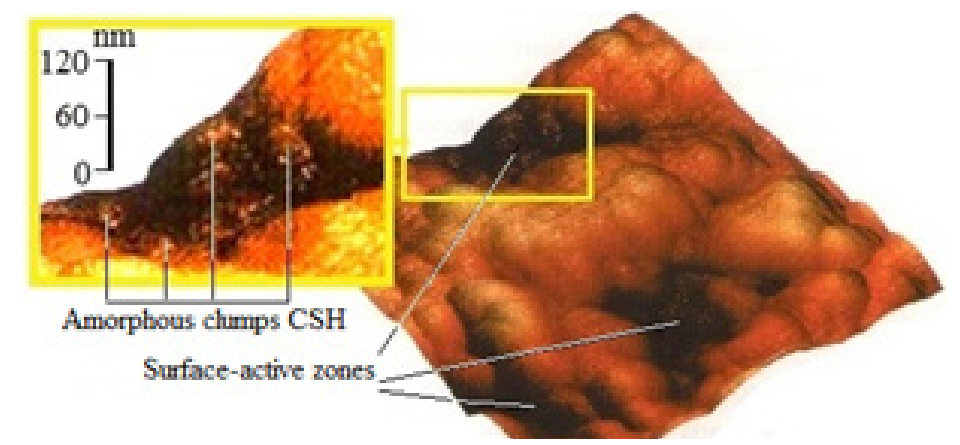

Figure 4: Morphology of neoplasms according to Bikbau [5]. 
The interaction of cement minerals with the water is happening by accumulating its own internal energy due to the formation of a meta-stable transition activated complex with its development, achievement of a critical energy level and decay at the boundary of the phases. The explosive destruction of Ca-Obonds in the structure of silicates, hydrogen and chemical bonds of adsorbed clusters, the appearance of active particles $\left(\mathrm{Ca}^{2+},\left(\mathrm{SiO}_{4}\right)^{4}\right.$, $\mathrm{H}_{3} \mathrm{O}^{+}, \mathrm{OH}^{-}$,etc.) and their fast-flowing interaction are the cause of the appearance on the surface of cement grains the adhesive "amorphized "layered-lumpy" structure with no practically signs of crystalline formations, which are characteristic for calcium hydroxide" [7] (Figure 4).

\section{Stages of the hydration process}

The hydration act completes by the rapid consumption by the minerals the portion of dipoles and the formation of the next activated complex with its subsequent development according to the noted scheme. This process at the initial stage (up to the maximum of heat release) at regular $\left(22 \pm 2{ }^{\circ} \mathrm{C}\right)$ hardening temperature

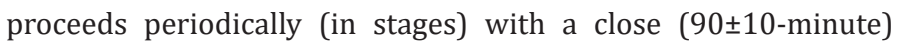
time interval (Figure 2) due to the small change in the size of the complexes and significant reserve of active moisture. As the chemical bonding of dipoles, the hydration of active centers and the decrease in the surface energy of cement particles happens, the induction stages naturally increase. The periodic release of calcium ions from the structure of cement minerals, the same nature of water consumption, the development of vacuum and the selforganization of the system are directly confirmed by the "wavy" or "stepped" nature of the change in many properties and phenomena (plastic strength, hardening temperature, chemical composition of pore fluid, speed of ultrasound, etc.).

\section{Discontinuity of hardening}

The stage chemical bonding of anhydrous cement minerals of water with its rapid consumption from pore space at the same time is accompanied by the development of a vacuum in the intergranular voids - the "driving force" of the formation and strengthening of the future cement stone and concretes. This phenomenon at the initial stage leads to a jump like solidification, recorded, for example, by characteristic fractures of the plastograms (Figure 2) and calorimetric curves (Figure 5). Consequently, this structure-forming feature, noted by Kind [1] in the early 30-ies of the last century [1] is not an anomaly, but a universal pattern characteristic of all clinker binders and materials based on them, of any compositions and consistencies that harden under any temperature-humidity conditions.

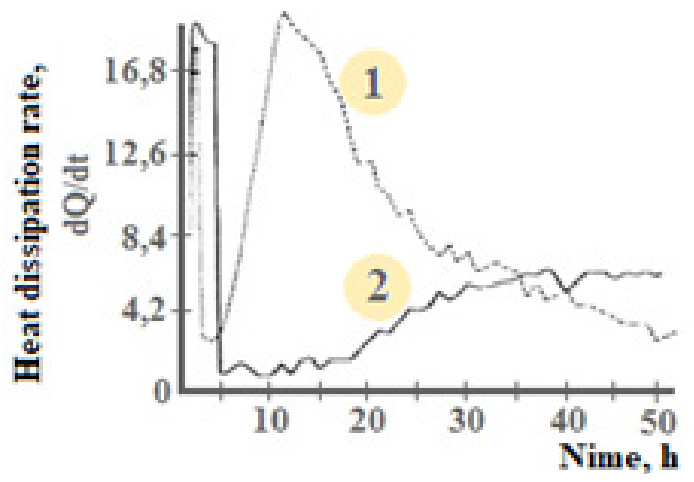

Figure 5: Heat dissipation rate of hydratable cement clinker Voskresensky plant [7].

1 - with the addition of $5 \%$ gypsum; 2 - without the addition of gypsum

\section{The essence of the induction period (and is it just single?)}

It would seems that a logical explanation of the nature of the induction period is the Western concept of the action of the "wetted surface" of cement particles due to the action of moisture on the surrounding fluid on the stored cement and the emergence on the grains of a hydrate layer $10 \ldots 100 \mathrm{~nm}$ thick, slowing down the authors' opinion $[8,9]$, hardening. However, this view contradicts the experiment. On the one hand, the induction period also occurs in a completely fresh (immediately after grinding) Portland cement. On the other hand, the induction period does not increase with a moderate degree of lethargy, but, on the contrary, it decreases, because a certain hydration stage of the process (dispersed adsorption of dipoles, formation of poly-molecular structures, their development) was carried out in the process of mass exchange phenomena during storage of cement. By adding water, we simply intensify the previously begun hydration process. This aspect is easily verified, for example, by the plastometric method - the first «jump» of structural strength on fresh cement is observed after $90 \pm 10 \mathrm{~min}$, after a six-month storage in a plastic tank (periodically opened for sampling) after $60 \pm 10 \mathrm{~min}$. In practice, this phenomenon is often encountered in the form of a relatively "quick grasp" of concrete on the old cement.

Usually refers to the initial, preceding the extraneous intensification of the hardening process time interval. As noted, during this hour and a half the development of an activated energy complex proceeds, reaching a critical level (the result of an increase in the $\xi$-potential of a double layer of particles is often detected by the initial expansion of the cement-water system) and decay. Outwardly, this period is unnoticeable (an inexpressive increase in structural strength, a lack of a thermal effect), its result is the interaction of elements, the release of calcium ions, the beginning of exothermia, the consumption of a portion of dipoles, the formation of a transitional energy composition and the self-organization of the system. Wherein induction period is no single. The whole stage 
of the existence of concrete as a building material is an alternation of induction time intervals and moments of process chemistry with indispensable destructive accompaniment.

\section{Influence of water-cement factory and aggregates}

The interaction of Portland cement with water flows in the dense area of the double electrical layer (circled in Figure 1), so that the water-cement factor and the presence of aggregate (sand, gravel, dispersed mineral additives and other chemically inert products) isn't effect on the quality of the process. The characteristic turning points of the kinetic curves of many (rheological, thermo chemical, electro physical) characteristics are observed at the same time in the dough and mixtures, i.e. they are the constant characteristic of this binder. Figuratively speaking, the process proceeds synchronously at the phase boundary, regardless of whether we placed cement grains in a container with water or a drop of water was put in a sack with cement. The time of the onset of the turning points can serve as a reliable reference point for a quantitative and qualitative assessment of the effect of various factors, including chemical additives, on the hardening of concrete.

\section{On the seizure of Portland cement}

The discrediting moment of the standard method for estimating the intensity of cement hardening by the so-called "setting time" is the mixing of the quantitative and qualitative aspects of hardening. Specific values of structural strength conditionally (!) are such gradual signs as "beginning" and "end" of the process. This led to confusion in concrete science due to the endowment for several factors functions alien to them (for example, anti-freeze additives potash according to GOST 24211- accelerating, despite its obviously slowing down effect). Consequently, the existing GOST 310.3 requires the correction in the use of the diagnostic method, based not on conditional indicators, but on the kinetics of properties that directly reflect the structure-forming process $[10,11]$.

\section{Superficiality of the process}

The hydration process is accompanied by a selective (incongruent according to Malinin [2]) dissolution of cement minerals with a predominant transition to the fluid of calcium ions (the conversion of which into lime determines the exothermy of the process) and the invariable location of the silicon-oxygen hydrolysis residues. The appearing hydrate product, in this regard, is characterized by a huge coupling with an array of cement grains and shielding properties, making incredible the deep spreading of the hydration front. In this connection, the idea of the formation of cement kernels of «relict grains» loses all its meaning. The surface nature of the process is confirmed by the absence of noticeable «dimensional metamorphosis» of cement grains after months, decades of normal and water hardening, and also after a complete loss of hydraulic activity as a result of repeated mixing, steaming and grinding of the product (Figure 6).

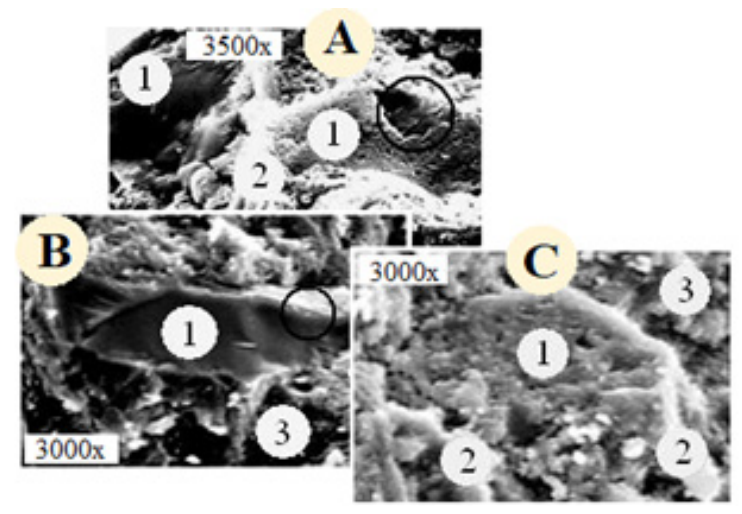

Figure 6: The structure of the three-month (A), 31-year (B) and fully hydrated (C) cement grain. 1-cement grain; 2-hydrated product; 3-intergranular emptiness

\section{The thickness of the hydrated layer}

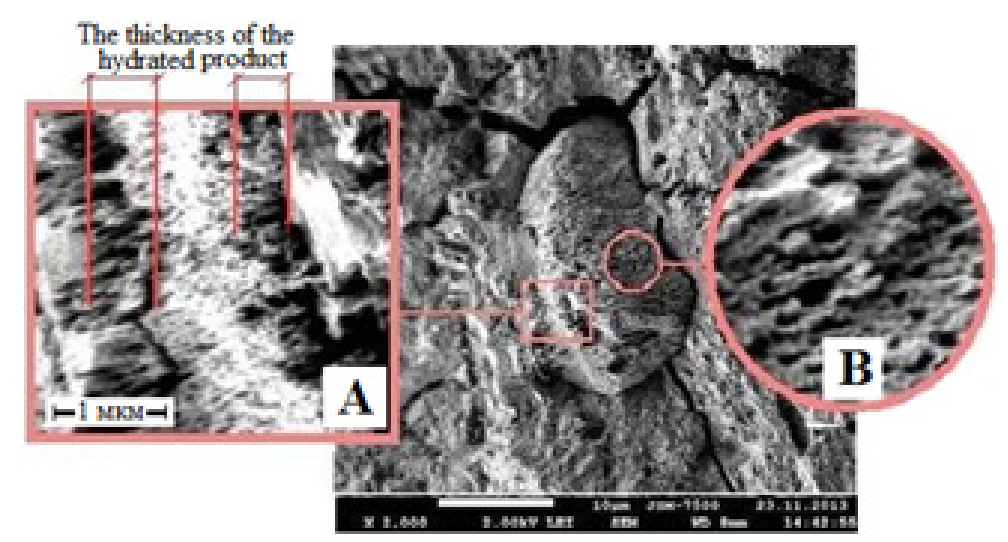

Figure 7: General appearance and fragments of the surface of cement water hardening grains. 
As a consequence of the previous position, the hydrated product throughout the solidification and the existence of the cement composite as a construction material is characterized by a practically constant and meager thickness of 1.0...1.5 $\mu \mathrm{m}$, which is confirmed by the experimental data obtained in recent years $[12,13]$. This aspect is clearly illustrated by the structural elements outlined in Figure 6 and the selected fragments of the grain of three-

\section{Degree of hydration of Portland cement}

year water aging (Figure 7), enabling a real quantitative assessment of this parameter. Considering the high porosity of the hydrated layer, it can be concluded that the penetration of the hydration front into the dense clinker array to a depth of a few parts of a micron. So, the trend of the relationship of the maximum use of the potential of cement with its complete dissolution, can hardly be recognized as wealthy.

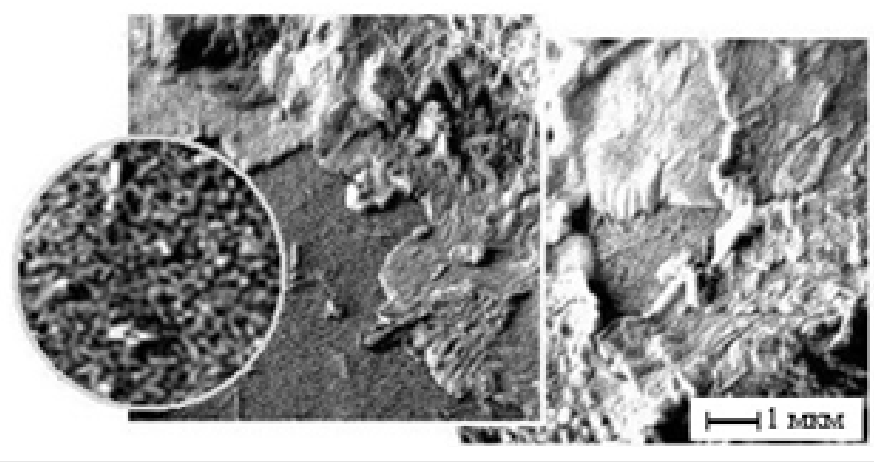

Figure 8: The structure of cement grains of 105 years of age.

The seeming contradiction between the constancy of the thickness of the hydrated layer and the steady increase in time the binder degree of hydration is quite simply solved taking into account the "constructive structure", the dynamics of the development of transition energy complexes, and the structural features of the residual surface-active zones (Figure 1). The uninterrupted energy activity of the latter, the inexhaustible supply of molecular-dispersed moisture of the environment, and the mass-exchange phenomena determine an inexhaustible in time hydration process, leading to consecutive hydration of the residual active centers for an indefinitely long time. In any case, the nonhydrated zones (with a diameter of $0.1 \mu \mathrm{m}$ or less) are clearly seen on 105-year-old cement grains (increased on Figure 8), which indicates a quite real hydration activity even at such a mature age.

\section{The structure of micro-concrete}

It is advisable to clarify certain provisions of the well-known term V.N. Jung "micro-concrete". Undoubtedly, cement stone is a highly heterogeneous product containing, along with other objects, many incompletely dispersed cement grains. However, these grains have not ceased to play an active role in hardening but are the determining factor a "large aggregate", a carrier of strength and other properties of micro-concrete. Optimization of the characteristics of the latter (as for ordinary concrete) is ensured by fractionation of the "filler"-the rational dispersed composition of the cement powder [1]. Effectively increasing the density of microconcrete with a structurally compatible mineral filler. At the same time, the size of the filler particles should be comparable with the dimensions of the intergranular voids (approximate specific surface $-5 \quad \ldots 10$ thousand $\mathrm{cm}^{2} / \mathrm{g}$ ). The larger and smaller size of the mineral additive worsens the result (due to the expansion of the cement grains in the first case and the appearance of additional problematic contact areas in the system- in the second).

\section{Morphology of calcium hydrosilicate}

Typically, the structure of C-S-H is considered in the form of petal, lenticular, scaly and similar structures, or by the result of the folding of thin tobermorite plates with the formation of channels for supplying water to the internal spheres of cement grains and transferring the dissolved products to the external fluid [3]. And although outwardly this picture corresponds to reality - the petallike elements border the cylindrical channels (Figure 3), causes a certain distrust in the crystallization path of their appearance. As a result of the stepwise reaction of the reagents, a banal periodic extrusion of hydro-silicate portions increasing in volume in the form of spherical formations up to $40 \mathrm{~nm}$ in size occurs in the pore space [14], which explains the "tubercle" (Figure 4) of the hydrated product. The pressure of amorphous hydro-silicate through the gaps of peculiar tightly bound "dipole spinnerets" determines the formation of fibrous-tubular structures (Figure 7).

\section{Saw-shaped character of hardening}

The hydration hardening of Portland cementand materials based on it (solutions, concretes) is a simultaneous, intimate and directly interconnected flow of positive (structure-forming) and negative (destructive) phenomena. Developing at the initial (plastic) stage in the system, the vacuum is the "driving force" that organizes the particles, which forms and strengthens the contact zones of microconcrete (concrete in general). The appearance of an increase in the volume of the solid phase of the hydrate at later stages, under the conditions of the existing structure of the composite, is the cause of internal tensile stresses, weakening of structural bonds, and dumping of strength. Under favorable circumstances, new portions of glutinous hydro-silicate "heal" microdefects, increase the density and strength of the stone, thereby determining the "sawtooth" [15] hardening character. Otherwise (for example, with a critical load), irreversible processes are possible, avalanche cracking and destruction of concrete «for no apparent reason». 


\section{Strength of concrete}

The strength of cement concrete is a dynamic, constantly changing (repeatedly increasing and decreasing) property. This dynamism is due to the mass-exchange processes of the cement composite with the environment, and the chemical reagents that are unlimited in time. Therefore, it is hardly justified to consider the strength of the bearing concrete as a property that determines the durability, reliability and the field of its application. The use of the strength index as a criterion for the implementation of certain actions (loading of columns of monolithic buildings by removing supporting supports of overlapping, transfer of stress of reinforcement to the structure, etc.) should be carried out with some care. Loading of concrete (reinforced concrete) follows structuralstable (induction) periods, and very undesirable results (Figure 9) can be obtained when performing this operation in destructive time intervals. In this regard, under the continuous control of loadbearing monolithic objects, there must be not only strength, but also dynamics, the nature of its development.

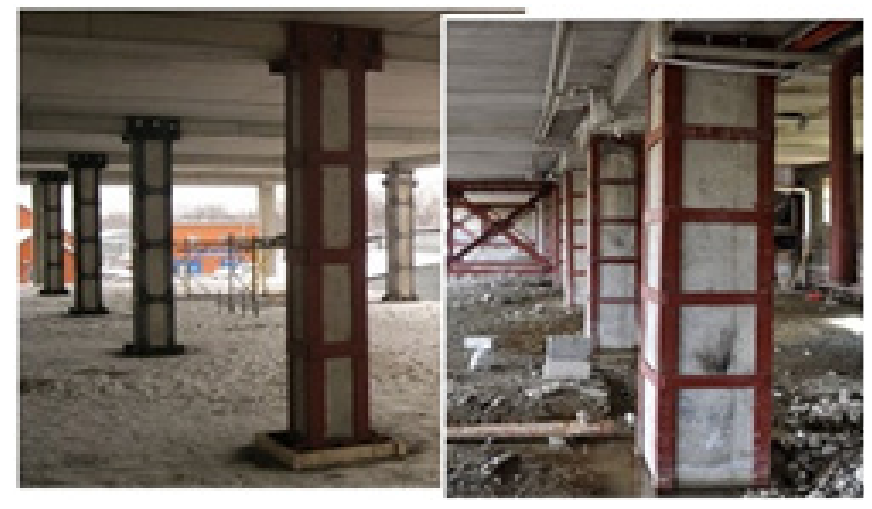

Figure 9: Strengthening of columns of multi-storey buildings.

\section{Contact area of concrete and reinforced concrete}

This structural element is the weakest, most vulnerable to aggressive environments, prone to primary destruction. The shrinking bonding system does not compress, but dissolves from the inert object (large and small filler, reinforcement, embedded parts), which becomes understandable, considering the displacement of the cement grains contacting with the inert surface into the intergranular void, i.e. from the surface of a chemically inert object to the area of maximum development of the vacuum. Hence the need for force (vibration, pressing, tamping) to harden concrete is necessary at rational times - the moments of cement grain cementing, the self-organization of the system. In production conditions, the time of vibro-activation is easily determined by the plastometric method according to the «bursts» of the curves constructed (Figure 2).

\section{Vitality and adaptability of concrete}

These characteristics of concrete are due to the above noted residual surface-active zones of hydrated clinker particles. The natural development of these zones in the process of mass exchange with the environment leads to periodic late hydration of cement with inevitable destruction. I. e. for concrete, as like as for a live organism, is inherent a peculiar temperature and structural pulsation (the absence of mass emergency situations is not a refutation of what has been said, but a confirmation of the asynchronous nature of the destructive phenomenon in the volume of concrete). Dangerous external (power, vibration, temperature, electromagnetic, etc.) effects, which activate adsorption-bound in micro-concrete water, capable of synchronizing the hydration and destructive processes on cement grains in an array of reinforced concrete construction with difficult predictable consequences.

\section{Operational reliability of concrete}

This fundamental criterion can be achieved by using a set of techniques that are good for solidification, which contribute to the completeness of the hydration process, the structural stability of micro-concrete. This is the enough amount of water, its activation by various (thermochemical, electrophysical, acoustic) methods, the use of a structurally compatible filler and highly disperse mineral filler, limiting of the use of novel polymeric hyper plasticizers and anti-freeze additives, vibro-activation support (optimal time for laying concrete mixes, repeated and cyclic vibrations), humidity conditions of hardening, protection of concrete from re-drying and marked in the previous the point of external influences.

\section{Conclusion}

In recent years, self-compacting high-strength concretes obtained by reducing water consumption, using hyper plasticizers and rational (continuous) granulometry of the mineral composition have become an enviable popularity. However, this at first glance, a positive trend, it can hardly be recognized as such for the following reasons. First, should remember the warning of the unconstrained concrete investigators: [16] "The use of high-strength concrete in bearing structures is necessary if the strength is achieved by combining low water-cement relation and additives of superplasticizers." We emphasize that in this case we are talking about superplasticizer, whose water-reducing capacity is incomparably lower than modern products, providing a $40-45 \%$ reduction in water consumption while maintaining high mobility. The deliberate reduction of one of the chemically active components is unlikely to contribute to the completeness and completeness of the hydration process in the technological stage (especially in the monolithic production of buildings and structures). 
In addition, the mechanism of plasticization (electrostatic dissociation of the cement system) enters into contradiction with the "driving force" of structure formation - a vacuum developing in intergranular voids. A combination of these factors determines the fact that the dumping of strength is more characteristic of high-strength concrete [17]. Cement dough, mortar and concrete mixes are staged (cyclically) self-compacting objects, by the nature of their hardening, requiring additional application of force in the plastic stage in rational terms (vibration, pressing, tamping). Cyclic vibrating is a factor that ensures compact packing of clinker particles, sealing of "adhesive layers" of micro-concrete, hardening of the contact zone "cement stone-aggregate (reinforcing elements)", extrusion of adsorption-bound water from non-hydrated zones, increase in the operational reliability of concretes.

To determine the time of densification, it is recommended to use the plastometric method, which consists in studying the kinetics of the plastic strength of a cement test hardening at specified temperature conditions and assigning the time of application of compaction to the characteristic turning points of the constructed plastogram. To duplicate data and capture a wider time interval, plastometric work should be carried out on a cement test with a multiple W / C value (for example, $0.26,0.28,0.30$ ).

\section{References}

1. Kind VA (1932) Chemical characterization of Portland cement. LM: Gosstroyizdat, pp. 3-4.

2. Malinina LA (1977) Thermal treatment of heavy concrete. Stroiizdat, Moscow, Russia, p. 160.

3. Shmitko EI, Krylova AV, Shatalova VV (2005) Chemistry of cement and astringents: Proc allowance-Voronezh. Arch Builds University, Voronezh, Russia, p. 164.

4. Lee FM (1961) Chemistry of cement and concrete. Gosstroyizdat, Moscow, Russia, p. 645.
5. Sychev MM (1974) Hardening of binders. Leningradskoe Dep, Stroyizdat, Leningrad, Russia, p. 80.

6. Skramtaev BG, Panfilova LI (1949) Investigation of the influence of vacuum in hardening cements. Proceedings of NII Cement, Promstroiizdat, Moscow, Russia 2: 6-8.

7. Sheikin AE (1974) Structure, strength and crack resistance of cement stone. Stroyizdat, Moscow, Russia, p. 191.

8. Bikbau M (2016) Nano-cements the future of the world cement industry and concrete technology. Technologies of concrete 3(4): 38-41.

9. Condo R, Daimon M (1976) Phase composition of the solidified cement test (main report). Sixth International Congress on Chemistry of Cement, Stroiizdat, Moscow, Russia 2: 244-257.

10. Scrivener KL, Juilland P, Monteiro PJM (2015) Advances in understanding hydration of Portland cement. Cement and Concrete Research 78(A): 3856.

11. Usherov Marshak AV (2010) Thermo kinetics as a direction of physicchemical analysis in concrete engineering. Concrete Technologies, 1112: 64-66.

12. Pshenichny GN, Kulikova AA (2013) On the diagnosis of Portland cements, or how much can you sit on Vicss needle? Technologies of concrete 3: 2729.

13. Klaus SP, Neubauer J, Goetz-Neunhoeffer F (2015) How to increase the hydration degree of CA-The influence of CA particle fineness. Cement and Concrete Research 67: 11-20.

14. Pshenichny GN (2016) On the hydration of Portland cement. Part 2 (stage-surface scheme). Technologies of concrete 7-8: 43-49.

15. (2011) News of nanotechnologies. Popular concrete science 1-2(38): 45-53.

16. Babkov VV, Polak AF, Komokhov PG (1988) Aspects of the long eternity of cement stone. Cement 3: 14-16.

17. Korovkin MO, Eroshkina NA (2016) Destructive processes in hardening high-strength concrete. Sb Theses of the reports of the international scientific and technical conference. High-strength cement concretes: Technologies, constructions, economics (VPB-2016), KSASU, Kazan, Russia, p. 42.

For possible submissions Click below: 